\title{
The role of kidney injury molecule- 1 in predicting cardiorenal syndrome type 1 after diuretic treatment
}

Adem Atici ${ }^{1}$, Samim Emet ${ }^{1}$, Ilkim Deniz Toprak², Ramazan Cakmak³, Murat Akarsu ${ }^{4}$, Tufan Tukek

\author{
${ }^{1}$ Department of Cardiology, Faculty of Medicine, Istanbul University, Istanbul, Turkey \\ 2Department of Internal Medicine, Istanbul Gaziosmanpasa Taksim Training and \\ Research Hospital, Istanbul, Turkey \\ ${ }^{3}$ Istanbul Department of Internal Medicine, Faculty of Medicine, Istanbul University, \\ Istanbul, Turkey \\ ${ }^{4}$ Department of Internal Medicine, Istanbul Okmeydani Training and Research \\ Hospital, Istanbul, Turkey
}

Submitted: 22 April 2019

Accepted: 12 July 2019

Arch Med Sci Atheroscler Dis 2019; 4: e208-e214

DOI: https://doi.org/10.5114/amsad.2019.87305

Copyright $\odot 2019$ Termedia \& Banach

\section{Abstract}

Introduction: Cardiorenal syndrome (CRS) is defined as acute or chronic dysfunction in the heart and kidney due to important interactions between the heart and kidney disease. The aim of this study was to evaluate prediction of CRS type 1 by measuring kidney injury molecule-1 (KIM-1) and to establish early diagnosis of acute kidney injury (AKI).

Material and methods: During 2015-2016, 146 patients who were admitted to the emergency service with acute decompensated HF were included in the study. We investigated urinary KIM-1 levels in 146 consecutive patients with decompensated heart failure before and after diuretic treatment. Statistical analyses were performed using the Statistical Package for the Social Sciences (SPSS - version 21.0)/Windows Statistical Software. $P$-values less than $<0.05$ were considered significant.

Results: There was a moderate negative correlation between the percentage change of creatinine values and the percentage change of KIM-1 values $(r=-0.357, p=0.016)$. There was no statistically significant relationship between KIM-1 and the development of CRS type $1(p=0.011)$.

Conclusions: No statistically significant relationship was observed between KIM-1 levels and the development of CRS type 1. In addition, there was no correlation between mortality in patients and KIM-1 values. It is thought that KIM-1 is not a potential prognostic indicator because renal tubular damage is only one of many factors in the pathophysiology of CRS type 1 and heart failure.

Key words: acute kidney injury, cardiorenal syndrome, kidney injury molecule-1.

\section{Introduction}

Cardiorenal syndrome (CRS) is defined as acute or chronic dysfunction of the heart and kidney due to important interactions between heart disease and kidney disease [1]. Generally, cardiac diseases are associated with the progression of renal disease and decrease in renal function [2]. Nowadays, the aging of society, the development of diagnostic procedures and treatment modalities have increased the incidence of CRS [3]. This situation leads to increased mortality, prolonged hospitalization, and more

\author{
Corresponding author: \\ Samim Emet \\ Department \\ of Cardiology \\ Faculty of Medicine \\ Istanbul University \\ 34390 Istanbul, Turkey \\ Phone: +90 5326652672 \\ E-mail: samim03@hotmail. \\ com
}


frequent admissions to the hospitals in patients with CRS $[4,5]$. There are 5 sub-types of CRS, and the incidence of type 1 CRS ranges between $24 \%$ and $45 \%$ [6]. CRS type 1 is known for worsening of kidney function in patients with acute decompensated heart failure. The diagnosis of acute kidney injury, or worsening of renal function, is based on acute increase in serum creatinine and/or the development of oliguria [7, 8].

Despite increased extracellular fluid volume, intravascular fluid volume is decreasing in most patients with CRS type 1 , which is especially apparent in diuretic usage. In order to maintain the intravascular fluid volume reduced by diuretic therapy, decreased renal perfusion because of the activation of the renin-angiotensin-aldosterone and sympathetic nervous systems, and activation of vasopressin; all of these leading to sodium and fluid retention. Increased angiotensin II production stimulates the release of many mediators that act as vasoconstrictors on kidney vessels [9]. These vasoconstrictor mediators play a role in the ischemic cascade of acute kidney injury (AKI) in decompensated heart failure $(\mathrm{HF})$, which results in further decreases in renal blood flow and glomerular filtration rates (GFR) [10]. Diuretics are important medications that control the volume status by reducing extracellular fluid in patients with HF. However, the patient population and diuretic dosage must be re-examined during treatment.

Early diagnosis can prevent AKI in patients with CRS type 1. Serum creatinine level used for diagnosis is insufficient for early detection because it begins to increase in 48-72 h after the onset of kidney injury [11]. At this point, new biomarkers are promising in early diagnosis. In particular, it is known that KIM-1 is more sensitive and specific for ischemic renal injury $[12,13]$. This molecule has been shown to be effective for the diagnosis of AKI in many clinical situations, such as coronary bypass surgery [14-16] and contrast-induced nephropathy [17].

In this study, while blood urea nitrogen (BUN) and serum creatinine levels were still within the reference ranges, KIM-1 levels of patients with acute decompensated HF were compared with kidney injury molecule-1 (KIM-1) levels of patients complicated with CRS. The aim of this study was to determine the effect of diuretic therapy on the pathophysiology of CRS and to evaluate the success of KIM-1 in showing early diagnosis of acute renal injury.

\section{Material and methods}

\section{Study population}

During 2015-2016, 146 patients who were admitted to the emergency service with acute decom- pensated HF were included in the study. Written informed consent was obtained from each patient before enrollment. These patients' diagnoses were confirmed using the diagnostic criteria of the European Society of Cardiology. Patients older than 18 years old who had decompensated HF symptoms and findings, with preserved ejection fraction or low ejection fraction, were included in the study. The exclusion criteria were the presence of acute myocardial infarction, cardiogenic shock, severe pulmonary disease, end-stage renal disease; receiving inotropic therapy; kidney or heart transplant recipients; previous stroke within the past six months; receiving chemotherapy; and planned intubation because of respiratory insufficiency.

Functional capacity and physical examination findings (edema, rales, jugular venous distension) of all patients were evaluated. The ejection fraction was evaluated using the Simpson method. GFR was calculated using the Cockcroft-Gault formula. An increase of serum creatinine level by more than $0.5 \mathrm{mg} / \mathrm{dl}$ or a $35 \%$ increase of serum creatinine over the baseline level were accepted as AKI.

Patients with a systolic blood pressure above $140 \mathrm{~mm} \mathrm{Hg}$, diastolic blood pressure above 90 $\mathrm{mm} \mathrm{Hg}$ in repetitive measurements or receiving antihypertensive therapy were considered as hypertensive. Patients with a fasting low-density lipoprotein (LDL) level above $130 \mathrm{mg} / \mathrm{dl}$ or taking statin therapy were considered as hypercholesterolemic. Patients with a fasting triglyceride level above $150 \mathrm{mg} / \mathrm{dl}$ or who were taking antilipidemic medication were considered as hypertriglyceridemic. Patients with a fasting blood glucose above $126 \mathrm{mg} / \mathrm{dl}$ or receiving antidiabetic treatment were considered as diabetic. Smoking status was identified as smokers and nonsmokers.

Written informed consent was obtained from each patient before enrollment in the study. The study was approved by the ethics committee

\section{Sample collection and measurements}

Patients who were followed up from the cardiology department for at least $72 \mathrm{~h}$ were included in the study. At least $7 \mathrm{ml}$ spot urine specimens were taken from the patients (two separate urine samples with 72-hour intervals). Urine samples were stored at $-80^{\circ} \mathrm{C}$ after centrifugation. Urinary KIM-1 levels were determined using an enzyme-linked immunosorbent assay (ELISA). The tests were repeated by a single investigator. The sensitivity and specificity were $96 \%$ in this method. Samples were studied according to the KIM-1 ELISA kit instructions. Biochemical and urine samples of the first and $72^{\text {nd }} h$ were quantitatively measured and recorded. Diuretic therapy (at least $100 \mathrm{mg}$ of furosemide per day) was then administered intravenously. 
All patients who were enrolled in the study underwent a comprehensive echocardiographic assessment using a Philips IE33 instrument and X5-1 transthoracic probe in the left lateral position (2-dimensional, M-mode, Doppler echocardiography) after at least 15 min rest. Echocardiography was performed following the standard images and techniques in the American and European Echocardiography Society (AEC) guidelines. All images were recorded digitally and then analyzed again.

Baseline and $72^{\text {nd }} \mathrm{h}$ serum creatinine values of the patients were measured. The patients were then divided into 2 groups according to whether their creatinine values were increasing (an increase of serum creatinine level by more than $0.5 \mathrm{mg} / \mathrm{dl}$ or a $35 \%$ increase of serum creatinine over the baseline level) or not.

\section{Statistical analysis}

Statistical analyses were performed using the Statistical Package for the Social Sciences (SPSS - version 21.0)/Windows Statistical Software. The Kolmogorov-Smirnov test was used to determine normal distribution. For quantitative data, the Mann-Whitney $U$ test was used to compare the two groups' parameters that did not show normal distribution, and the independent samples $t$-test was used to evaluate parameters with normal distribution. The paired $t$ test was used for normal distribution and the Wilcoxon T test was used for non-normal distribution. Pearson's or Spearman correlation coefficients were used where appropriate. $P$-values less than $<0.05$ were considered significant. Also, descriptive statistical methods (mean, standard deviation, median, frequency, ratio, minimum, maximum) were used.

\section{Results}

\section{Demographic features}

The clinical demographic characteristics of the 146 patients included in the study are shown in Table I. Most patients with chronic HF, who were followed up for an average of $9.16 \pm 4.5$ years, had ischemic heart disease and atrial fibrillation, and most of them were men $(62 \%)$. Half of the patients were using angiotensin-converting enzyme inhibitors/ angiotensin receptor blockers and spironolactone in addition to loop diuretics. The functional capacities of all patients were assessed as more than class 2 according to the New York Heart Association (NYHA) classification. Left ventricular ejection fractions were lower than $40 \%$ in half of the patients and higher than $50 \%$ in the other half. eGFR was lower than $60 \mathrm{ml} / \mathrm{min} / 1.73 \mathrm{~m}^{2}$ in $55 \%(n=80)$ of patients.

The baseline creatinine value was measured as $1.33 \pm 0.35 \mathrm{mg} / \mathrm{dl}$. AKI was detected in 90 (62\%) patients. Although baseline KIM-1 levels were higher in patients with increased creatinine levels, no statistically significant difference was found in KIM-1 levels between them and patients without AKI $(2.16 \pm 0.40 / 1.98 \pm 0.28, p=0.117)$ (Figure 1).

Table I. Basic characteristics of the 146 patients with acute decompensated heart failure

\begin{tabular}{|c|c|}
\hline Parameter & Result \\
\hline Number of patients & 146 \\
\hline Age [years] & $71.02 \pm 10.17$ \\
\hline Sex (female), $n(\%)$ & $56(38)$ \\
\hline $\mathrm{HT}, n(\%)$ & $90(62)$ \\
\hline $\mathrm{DM}, n(\%)$ & $73(50)$ \\
\hline CAD, $n(\%)$ & $83(57)$ \\
\hline CVE, $n(\%)$ & $11(8)$ \\
\hline Smoking, $n(\%)$ & $48(33)$ \\
\hline Baseline creatinine $[\mathrm{mg} / \mathrm{dl}]$ & $1.33 \pm 0.35$ \\
\hline Control creatinine [mg/dl] & $1.50 \pm 0.50$ \\
\hline $\mathrm{eGFR}\left[\mathrm{ml} / \mathrm{min} / 1.73 \mathrm{~m}^{2}\right]$ & $59.24 \pm 19.03$ \\
\hline $\mathrm{SBP}[\mathrm{mm} \mathrm{Hg}]$ & $121.24 \pm 20.54$ \\
\hline $\mathrm{DBP}[\mathrm{mm} \mathrm{Hg}]$ & $67.86 \pm 11.11$ \\
\hline LVEF (\%) & $45.96 \pm 16.30$ \\
\hline LVEF ( $\geq 50 \%), n(\%)$ & $71(49)$ \\
\hline NYHA III/IV, n (\%) & $23(51.1) / 22(48.9)$ \\
\hline Baseline KIM-1 [ng/ml] & $2.0963 \pm 0.3684$ \\
\hline Follow-up KIM-1 [ng/ml] & $2.0288 \pm 0.5799$ \\
\hline Pro BNP $[\mathrm{pg} / \mathrm{ml}]$ & $4460(3012-11133)$ \\
\hline \multicolumn{2}{|l|}{ ProBNP [pg/ml], $n(\%):$} \\
\hline$\leq 4460 \mathrm{pg} / \mathrm{ml}$ & $75(52)$ \\
\hline$>4460 \mathrm{pg} / \mathrm{ml}$ & $71(48)$ \\
\hline \multicolumn{2}{|l|}{ Medication $n(\%)$ : } \\
\hline ACEI/ARB & $74(51)$ \\
\hline Spironolactone & $61(42)$ \\
\hline Statin & $37(26)$ \\
\hline Thiazide diuretics & $37(26)$ \\
\hline Loop diuretics & $146(100)$ \\
\hline OAD & $51(35)$ \\
\hline
\end{tabular}

$A C E I$ - angiotensin converting enzyme inhibitors, $A R B$ - angiotensin receptor blockers, $B N P$ - brain natriuretic peptide, $C A D$ - coronary artery disease, CVE - cerebrovascular event, DM - diabetes mellitus, DBP - diastolic blood pressure, EF- ejection fraction, eGFR - estimated glomerular filtration rate, HT - hypertension, KIM-1 kidney injury molecule 1, NYHA - New York Heart Association, OAD - oral antidiabetic, SBP - systolic blood pressure. 


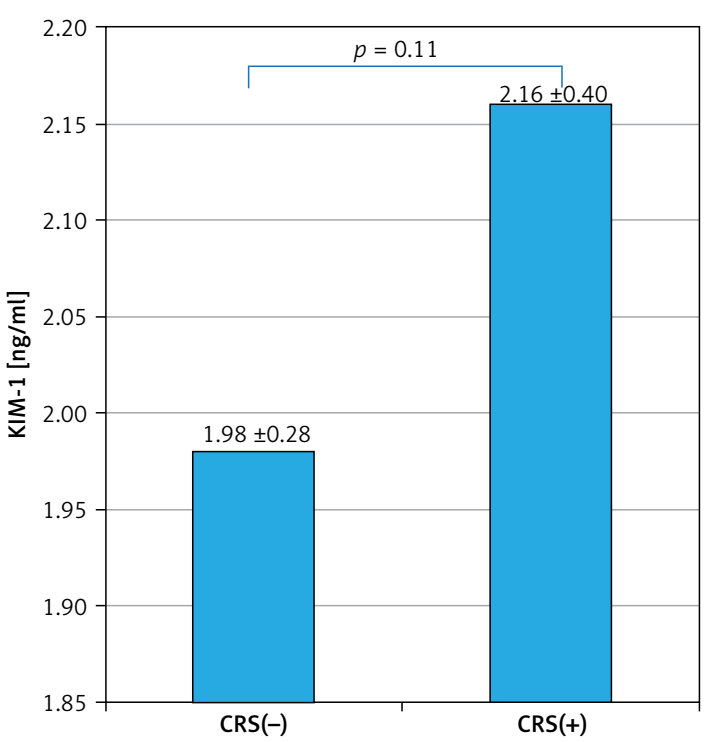

Figure 1. Comparison of baseline KIM-1 values in patients with and without cardiorenal syndrome

The baseline and $72^{\text {nd }} \mathrm{h}$ creatinine values were measured. After the patients were divided according to whether their creatinine values were increasing or not, a statistically significant increase was found in the KIM-1 levels of patients without AKI $(1.98 \pm 0.28 / 2.33 \pm 0.61 ; p=0.05)$. In the group with increased creatinine values, there was a statistically significant decrease in $\mathrm{KIM}-1$ values $(2.16 \pm 0.40 / 1.84 \pm 0.47 ; p=0.018)$. At the same time, there was a moderate negative correlation between the percentage change of creatinine values and the percentage change of KIM-1 values $(r=-0.357, p=0.016)$ (Figure 2).

Patients were divided into two groups according to ejection fraction as preserved and low ejection fraction. There was no statistically significant

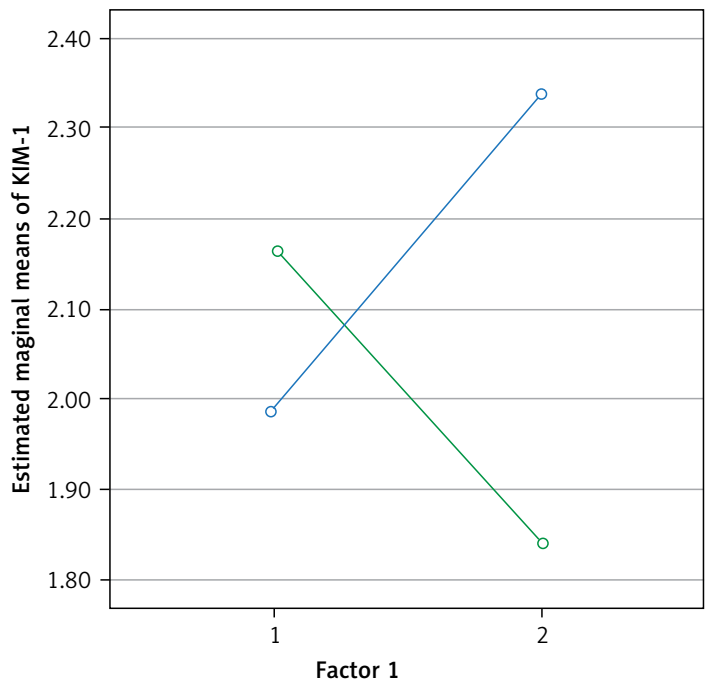

Figure 2. Relationship between creatinine levels and KIM-1 (The blue bar represents creatinine and the green bar represents $\mathrm{KIM}-1$ ) difference between baseline creatinine values $(1.32 \pm 0.34 / 1.33 \pm 0.38 ; p=0.874)$ and the creatinine values of the $3^{\text {rd }}$ day $(1.51 \pm 0.53 / 1.49 \pm 0.47$; $p=0.889)$ in the 2 groups. Although it is not statistically significant, baseline KIM-1 levels were higher in patients with normal ejection fraction (1.98 $\pm 0.32 / 2.06 \pm 0.74 ; p=0.637)$. As a result, patients with preserved or low ejection fraction were divided into two groups; there was no statistically significant differences in creatinine levels between these groups. In addition, there was no statistically significant difference between the KIM-1 levels of these 2 groups.

We researched whether there was a relationship between baseline eGFR values and baseline $\mathrm{KIM}-1$ values; there was no correlation between them $(r=0.08, p=0.957)$. Patients were divided into 2 groups according to their eGFR values, lower and higher than $60 \mathrm{ml} / \mathrm{min} / 1.73 \mathrm{~m}^{2}$. No association was detected between KIM-1 levels and baseline values of eGFR (2.09 $\pm 0.31,2.09 \pm 0.40$; $p 1=0.966$ ).

Patients were divided into two groups depending on the dosage they received over a 72-hour period. The limit furosemide dosage was determined as $320 \mathrm{mg}$ per $72 \mathrm{~h}$. There was no significant difference between the baseline values of $\mathrm{KIM}-1$ in these two groups $(2.09 \pm 0.34 / 2.06 \pm 0.44$; $p=0.846)$. After diuretic treatment, there was no statistically significant difference in follow-up KIM-1 values between these two groups either (2.05 $\pm 0.64,2.04 \pm 0.33 ; p=0.995)$. As a result, there was no difference between the patients' baseline, follow-up, and delta KIM-1 values or diuretic doses (Figure 3).

Similarly, patients were divided into two groups depending on the median levels of proBNP. When the groups were created, the cut-off level of proBNP was determined as $4460 \mathrm{pg} / \mathrm{ml}$. Both groups' baseline creatinine, baseline $\mathrm{KIM}-1$, follow-up creatinine, and follow-up KIM-1 levels were compared separately. There was no statistically significant dif-

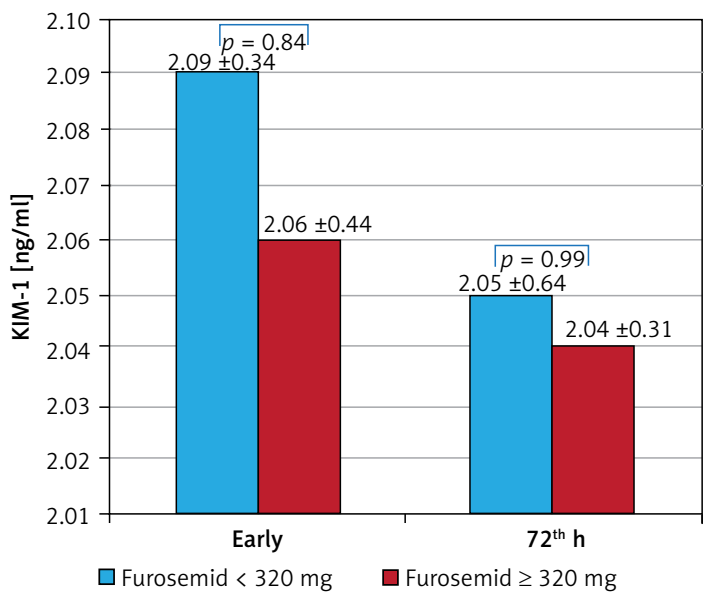

Figure 3. Effect of diuretic dose on KIM-1 
ference between baseline KIM-1 (2.06 $\pm 0.30 / 2.12$ $\pm 0.42 ; p=0.608)$ and follow-up KIM-1 (1.96 $\pm 0.67 /$ $2.09 \pm 0.47 ; p=0.465)$. Also, there was no significant difference between baseline creatinine (1.26 $\pm 0.34 / 1.39 \pm 0.36 ; p=0.261)$ and follow-up creatinine $(1.43 \pm 0.48 / 1.58 \pm 0.52 ; p=0.306)$.

The patients were divided into 2 groups depending on their functional capacity classes. The functional capacities of the groups were determined as class 3 and 4 . There was no statistically significant difference between baseline $\mathrm{KIM}-1$ $(2.09 \pm 0.34 / 2.09 \pm 0.40 ; p=0.950)$ and control KIM-1 $(2.05 \pm 0.60 / 1.99 \pm 0.56 ; p=0.740)$ between these two groups.

Finally, the relationship between mortality and KIM-1 was evaluated and no statistically significant difference was found between baseline KIM-1 $(2.08 \pm 0.42 / 2.12 \pm 0.30 ; p=0.760)$ and follow-up KIM-1 $(2.10 \pm 0.65 / 1.86 \pm 0.49 ; p=0.231)$ in patients who survived and those who died.

\section{Discussion}

We assessed the novel urinary biomarker KIM-1 for the diagnosis of AKI, because of the insufficiency of the creatinine molecule in detecting the early stages of the development of CRS in patients with decompensated HF. Unlike the studies by Kaddourah et al. [18] and Jungbauer [19], our results did not support the superiority of KIM-1 in predicting CRS in decompensated HF. No relationship was found between KIM-1 and ejection fraction or NYHA classes ( $p 1=0.412, p 2=0.950)$. This may be related to the use of different methodologies and different patient groups in these studies. Also, the reflections in KIM-1 levels may be different from the other studies in patients with decompensated HF due to the dysfunction of compensation mechanisms. In addition, the predisposing factor that causes decompensated $\mathrm{HF}$ is different in many patients, so it is not known how they affect the synthesis of KIM-1.

Although baseline KIM-1 levels were higher in patients progressing to CRS than in patients without CRS, the difference was not statistically significant ( $p=0.117$ ). KIM-1 is more sensitive and specific than creatinine in detecting renal azotemia. However, in prerenal azotemia (more than $48 \mathrm{~h}$ ), the increase of KIM-1 levels was not as large as the increase of creatinine. In our study, it is unclear whether this increase in serum creatinine was due to a real renal tubular injury. Sometimes it may be difficult to determine the type of CRS because the time, cause, status, or persistence of the interaction between damage and signs/symptoms in organs is unknown $[3,20]$. Another situation is that the patient can switch between the subtypes of CRS during the course of the illness $[1,21]$. Considering that 5 subtypes of CRS are present, it is expected that the increase of creatinine may be caused by ischemic renal injury and other situations. Therefore, it would not be appropriate to completely ignore the predictive value of $\mathrm{KIM}-1$ in our patients who progressed to CRS.

In our study, there was no difference between KIM-1 levels in patients with increased creatinine and patients with decreased creatinine. In 2016, Chen et al. assessed the level of urinary KIM-1 in patients with acute decompensated $\mathrm{HF}$ as we did [22]. In our study, KIM-1 levels were found to be $1.98 \mathrm{ng} / \mathrm{ml}$ in patients without AKI and $2.16 \mathrm{ng} /$ $\mathrm{ml}$ in patients with AKI. The value of KIM-1 in our study was similar to that of patients with ischemic AKI reported in 2002 by Han et al. $(2.92 \mathrm{ng} / \mathrm{ml})$ [23].

We also found an inverse relationship between KIM-1 and creatinine in our study. As follows, we observed that when KIM-1 levels were decreasing, creatinine levels were increasing significantly $(p=0.018)$. This relationship between creatinine and KIM-1 may be explained by the increased release of KIM-1, which probably had an apoptotic feature, from damaged proximal tubule epithelial cells and the reduction of cell number by stimulating apoptosis in these cells. Although the synthesis of KIM-1 increases, the decrease in the number of cells expressing this molecule may explain this situation. Despite the fact that creatinine is constantly present in the circulation with no change in its production and a decrease in its excretion in renal injury. On the other hand, KIM-1 is synthesized in renal tubules. So, the inverse relationship between creatinine and KIM-1 can be explained by the decrease in production of KIM-1 in renal injury.

In a recent study in 2014 by Coca et al., urinary levels of KIM-1 in the immediate postoperative coronary artery bypass graft period provided additional prognostic information for 3-year mortality risk in patients with and without clinical AKI [24]. However, the relationship between mortality and KIM-1 was evaluated and no statistically significant difference was found between baseline KIM-1 and follow-up KIM-1 in patients who survived and those who died in our study.

If treatment approaches are considered, it is predicted that the tubular marker will also be important in the management of the patient's fluid balance and diuretic treatment. A strong relationship between high-dose diuretic therapy and KIM-1 levels was found in the study by Jungbauer et al. in 2011 [19]. By contrast, there was no significant relationship between diuretic doses and KIM-1 levels in our study ( $p 1=0.846, p 2=0.995)$. The effect of diuretic-induced fluid loss on GFR is altered in patients with HF. In some patients, the decrease in cardiac filling pressures reduces cardiac output and reduces renal perfusion, and finally GFR decreases [25]. However, GFR is not af- 
fected in some patients but it causes decreased serum creatinine levels in others. There are two situations that may explain this situation: one is the initiation of diuretic treatment when we consider baseline creatinine levels, symptoms, and findings; and the dosage reduction as soon as the symptoms are normalized and medication is discontinued before ischemia occurs in the kidney. Secondly, CRS may be due to the presence of many physiopathologic processes. Different physiopathologies for each patient, decompensation of the patient (because of the inability of the body's compensatory mechanisms), and older age may also affect the release patterns of KIM-1 and similar molecules. All of these factors may reduce the sensitivity and specificity of KIM-1. However, we cannot rule out the presence of an as yet undefined tubular protein that is probably secreted after activation of KIM-1, which directly affects the development of cardiac dysfunction and subsequent HF. This should be reviewed in further studies.

As a result, in patients with HF, KIM-1 is superior to conventional methods (e.g., creatinine, urea) that assess subclinical renal injury. Unfortunately, our results do not support the superiority of KIM-1 in predicting type 1 CRS in decompensated HF.

Our results should be interpreted in view of some study limitations. Firstly, the main limitation of the study is that it was a single-center study and had a limited sample size. Secondly, it should be noted that our population was predominantly male $(62 \%)$ and elderly (mean age: $71.02 \pm 10.17$ years) with only acute HF and NYHA class III or IV symptoms. Therefore, the results may not correlate with younger and female patients with chronic HF and NYHA class I or II symptoms. Thirdly, information about the normal range of KIM-1 was determined according to previous studies because healthy volunteers were not included in the study. Accordingly, our findings should be considered as hypothesis-generating and require confirmation.

In conclusion, this study, in a cohort design, investigated the role of the new renal biomarker KIM-1 in the prediction of CRS type 1 after diuretic therapy in patients with decompensated HF. No statistically significant relationship was observed between KIM-1 levels and the development of CRS type 1 . In addition, there was no correlation between mortality and KIM-1 levels. It is thought that KIM-1 is not a potential prognostic indicator because renal tubular damage is only one of many factors in the pathophysiology of CRS type 1 and HF.

Further randomized controlled studies are needed to understand new biomarkers that have been studied in a single center, in small patient groups, and mostly with frozen specimens before routine usage. Although serum creatinine has dis- advantages, it maintains its diagnostic and follow-up position due to its practicality, simplicity, and repeatability.

\section{Conflict of interest}

The authors declare no conflict of interest.

\section{References}

1. Ronco C, House AA, Haapio M. Cardiorenal syndrome: refining the definition of a complex symbiosis gone wrong. Intensive Care Med 2008; 34: 957-62.

2. Elsayed EF, Tighiouart H, Griffith J, et al. Cardiovascular disease and subsequent kidney disease. Arch Intern Med 2007; 167: 1130-6.

3. Fabbian F, Pala M, De Giorgi A, et al. Clinical features of cardio-renal syndrome in a cohort of consecutive patients admitted to an internal medicine ward. Open Cardiovasc Med J 2011; 5: 220-5.

4. Damman K, Jaarsma T, Voors AA, Navis G, Hillege HL, van Veldhuisen DJ. Both in- and out-hospital worsening of renal function predict outcome in patients with heart failure: results from the Coordinating Study Evaluating Outcome of Advising and Counseling in Heart Failure (COACH). Eur J Heart Fail 2009; 11: 847-54.

5. Smith GL, Lichtman JH, Bracken MB, et al. Renal impairment and outcomes in heart failure: systematic review and meta-analysis. J Am Coll Cardiol 2006; 47: 1987-96.

6. Chittineni H, Miyawaki N, Gulipelli S, Fishbane S. Risk for acute renal failure in patients hospitalized for decompensated congestive heart failure. Am J Nephrol 2007; 27: 55-62.

7. Spanaus KS, Kronenberg F, Ritz E, et al. B-type natriuretic peptide concentrations predict the progression of nondiabetic chronic kidney disease: the mild-to-moderate kidney disease study. Clin Chem 2007; 53: 1264-72.

8. Kelly KJ. Acute renal failure: much more than a kidney disease. Semin Nephrol 2006; 26: 105-13.

9. Neuhofer W, Pittrow D. Role of endothelin and endothelin receptor antagonists in renal disease. Eur J Clin Investig 2006; 36 Supl. 3: 78-88.

10. Smilde TD, Damman K, van der Harst P, et al. Differential associations between renal function and "modifiable" risk factors in patients with chronic heart failure. Clin Res Cardiol 2009; 98: 121-9.

11. Perrone RD, Madias NE, Levey AS. Serum creatinine as an index of renal function: new insights into old concepts. Clin Chem 1992; 38: 1933-53.

12. Honore PM, Joannes-Boyau O, Boer W. The early biomarker of acute kidney injury: in search of the Holy Grail. Intens Care Med 2007; 33: 1866-8.

13. Mishra J, Ma Q, Prada A, et al. Identification of neutrophil gelatinase-associated lipocalin as a novel early urinary biomarker for ischemic renal injury. J Am Soc Nephrol 2003; 14: 2534-43.

14. Sirota JC, Walcher A, Faubel S, et al. Urine IL-18, NGAL, IL-8 and serum IL-8 are biomarkers of acute kidney injury following liver transplantation. BMC Nephrol 2013; 14: 17.

15. Mishra J, Dent C, Tarabishi R, et al. Neutrophil gelatinase-associated lipocalin (NGAL) as a biomarker for acute renal injury after cardiac surgery. Lancet 2005; 365: 1231-8.

16. Parikh CR, Devarajan P, Zappitelli M, et al. Postoperative biomarkers predict acute kidney injury and poor out- 
comes after pediatric cardiac surgery. J Am Soc Nephrol 2011; 22: 1737-47.

17. Gul CB, Gullulu M, Oral B, et al. Urinary IL-18: a marker of contrast-induced nephropathy following percutaneous coronary intervention? Clin Biochem 2008; 41 : 544-7.

18. Kaddourah A, Goldstein SL, Basu R, et al. Novel urinary tubular injury markers reveal an evidence of underlying kidney injury in children with reduced left ventricular systolic function: a pilot study. Pediatr Nephrol 2016; 31: 1637-45.

19. Jungbauer CG, Birner C, Jung B, et al. Kidney injury molecule-1 and $\mathrm{N}$-acetyl-beta-d-glucosaminidase in chronic heart failure: possible biomarkers of cardiorenal syndrome. Eur J Heart Fail 2011; 13: 1104-10.

20. lyngkaran P, Schneider H, Devarajan P, Anavekar N, Krum H, Ronco C. Cardio-renal syndrome: new perspective in diagnostics. Semin Nephrol 2012; 32: 3-17.

21. Cruz DN. Cardiorenal syndrome in critical care: the acute cardiorenal and renocardiac syndromes. Adv Chronic Kidney Dis 2013; 20: 56-66.

22. Chen C, Yang X, Lei Y, et al. Urinary biomarkers at the time of AKI diagnosis as predictors of progression of AKI among patients with acute cardiorenal syndrome. Clin J Am Soc Nephrol 2016; 11: 1536-44.

23. Han WK, Bailly V, Abichandani R, Thadhani R, Bonventre JV. Kidney injury molecule-1 (KIM-1): a novel biomarker for human renal proximal tubule injury. Kidney Int 2002; 62: 237-44.

24. Coca SG, Garg AX, Thiessen-Philbrook H, et al. Urinary biomarkers of AKI and mortality 3 years after cardiac surgery. J Am Soc Nephrol 2013; 25: 1063-71.

25. Stampfer M, Epstein SE, Beiser GD, Braunwald E. Hemodynamic effects of diuresis at rest and during intense upright exercise in patients with impaired cardiac function. Circulation 1968; 37: 900-11. 